Kocaeli Journal of Science and Engineering

\title{
Fault Maintenance and Repair Application Based on Augmented Reality Technology in Electrical Distribution Systems
}

\author{
Gokmen HASANCEBI ${ }^{1}$ (D) , E. Mustafa YEGIN ${ }^{2}$ (D) , Korhan KARAARSLAN ${ }^{3, *}$ (iD) \\ ${ }^{1}$ Sakarya Electricity Distribution Company, Kocaeli, 41310, Turkey, ORCID: 0000-0002-2799-3052 \\ ${ }^{2}$ Department of Electrical Engineering, Kocaeli University, Kocaeli, 41380, Turkey, ORCID: 0000-0002-1665-3132 \\ ${ }^{3}$ Department of Electrical Engineering, Kocaeli University, Kocaeli, 41380, Turkey, ORCID: 0000-0001-7109-3636
}

\begin{tabular}{l} 
Article Info \\
\hline Research paper \\
Received : February 3, 2021 \\
Accepted : March 11, 2021
\end{tabular}

Keywords

Augmented Reality

Fault Scenario

Object Recognition

SCADA

\begin{abstract}
This study presents an AR technology-based software that will work on mobile platforms and the pilot application of this software to solve the possible problems that may be experienced other than routine maintenance activities on protection/control panels and devices in SCADA centers. AR is essentially an information technology that provides the ability to present digital data and images in the same environment as our real world through its visualization, instruction/guidance, and interaction capabilities. Object recognition methods, necessary software, and infrastructure requirements are mentioned in this study in order to benefit from these advantages of AR technology correctly. Different scenarios have been created by using AR for possible failures in automation panels in a distribution center with a telemetry system. Finally, an exemplary scenario in the study is realized in a distribution center located in the SEDAŞ area of responsibility.
\end{abstract}

\section{Introduction}

The continuous increase in demand for electrical energy is an important part of our lives today. Thus, the amount of energy consumed per capita is shown as an indicator of every country's development level. The increase in energy demand has created a more reliable and high-quality energy concept known as "power quality" in electrical engineering. To meet the continuous and highquality energy demand of consumers, it is necessary to take action quickly and safely against possible problems in electrical energy systems. In this context, it is thought that the use of information technologies to resolve problems rapidly in SCADA systems and to improve the quality of service for electricity distribution companies will have a positive impact on the maintenance/repair (MR) processes.

There is an essential inconsistency between the rich digital data presented to us and the physical world in which we apply. Although, the physical world is three-dimensional (3D), digital data affects our decisions but practices are happening in two dimensions. The difference in dimensions must be mentally eliminated, and turning the digital data into the 3D real world. Augmented Reality (AR) technology can eliminate the obligation because it has the ability to present digital data and images in the same environment like the real world [1].

In the future, it is inevitable that all kinds of structures and industries, from educational institutions to social institutions, will be affected by AR technology. AR will change our decision mechanisms and learning styles. In addition, AR will change the way companies train their employees, deliver services, design their products, manage value chains, and compete. AR is an information technology that has visualization, instruction/guidance, and interaction capabilities that can make certain changes.

Through the visualization feature, it is ensured that the interior parts of a product, which are impossible to see under normal conditions, are made visible. For example, a medical device company that converts vein temperature data into images on the patient's skin reports a $350 \%$ increase in the

\footnotetext{
* Corresponding Author: korhan.karaarslan@kocaeli.edu.tr
} 
rate of venipuncture on the first attempt [2].

The instruction, training, and guidance processes required to increase productivity and service quality, ensure occupational safety, and take quick action are redefined with AR. It is difficult to follow written instructions/directions during the manufacturing phase. Training sessions are expensive and time-consuming, as well as creating space and equipment is required. AR can overcome such problems in a real physical environment with an array of real-time visual instructions. Boeing Company used AR technology in training for a process that included 50 step assembly drawings and found that training time was reduced by $30 \%$ compared to traditional training time [3]. In addition, ARbased devices can send images to an expert who is not in the field, and thus the performance of the personnel in the field can be increased and costs can be saved.

AR technology offers a virtual control panel that is projected directly on the product, instead of using physical control elements such as buttons, valves, and touch screens. Thus, it enables users to manage products remotely. With AR-based devices, it is possible to control the product through hand gestures and voice commands.

Although initially AR technology was used in limited areas, it is used in many areas in a short time in parallel with technological developments (increase in processor capacities and image quality in cameras). In addition, the availability of end-user hardware such as wearable AR systems and smart glasses (Magic Leap, Microsoft HoloLens,), has increased this usage much faster. Currently, the areas where AR technology is mostly used are games, educational applications, advertising and marketing, architectural and construction applications, health, routing and travel applications, military equipment, and industry 4.0 applications [4-14].

The electricity sector is leading those industries that are not yet using AR technology enough. Because of the literature review, it has been determined that the applications of AR technology in the electric energy sector are also limited. Virtual Reality (VR) applications were included in the first studies [15-18]. While digital data are superimposed on the real world in AR applications, the real world is simulated in a computer environment in VR applications. An application showing the appearance of the basic elements in a transformer substation, the functions of these elements, and how to perform the switching operations were conducted by [15] for the purpose of personnel training. Another study describes how VR technology can be used to visualize electrical power systems and how to make professional educational activities more effective. For this purpose, a study was conducted for expert staff involved in the diagnosis, MR of complex machines such as transformers and generators [16]. In 2013, an application developed for a transformer center provided the opportunity for visitors to navigate in a virtual environment. In addition, the user was enabled to perform real-time operations with real data obtained from the field [17]. With a VR application of electrical tests performed in substations, the aim of using this technique was to increase the number of electrical technicians and train the undergraduate students [18].

In the following years, VR applications were replaced with AR applications. In a study where AR technology is used in the electricity energy sector, a typical $220 \mathrm{kV}$ transformer substation in Shanghai Electricity Grid was selected as a pilot application. AR models of primary and secondary equipment were created to improve the operations and MR training [19]. In 2017, the French electricity distribution company Edenis implemented an AR application consisting of a series of instructions in order to facilitate the use of low voltage control panels and medium voltage devices as well as to reduce possible errors. The same distribution company also implemented an application that enables the field staff to easily locate the fault in underground cables using smart glasses [20, 21]. In another study in which AR technology was used, a mobile application was made to determine and monitor the amount of sag in overhead transmission and distribution lines [22].

As can be seen from the results of the literature review, AR technology, which has a limited number of applications in electrical energy systems, is also underutilized in electrical energy systems in our country. In order to take an advantage of AR technology, a research and development project was carried out in a distribution substation located in the area of responsibility of Sakarya Electricity Distribution Corporation (SEDAS). This project, for the use of AR technology, forms the basis of the proposed study. In the project, the aim is to develop an AR technology-based application on mobile platforms to be used in MR operations of SCADA systems and to support on-field technical teams with quick diagnosis using this application.

The following part of the study mentions the methods of object recognition, the necessary needs for software and infrastructure, which will be used in the AR application. The third section contains an example scenario for an AR application designed for possible failures on the automation panels of the remote terminal unit (RTU) and the data concentrator unit (DCU) belonging to the distribution substation. Achievements with the application are described in the fourth part.

\section{Materials and Methods}

Some applications are relatively simple according to complex and instruction-based AR applications, such as furniture and decoration applications that allow you to 
visualize objects in different configurations and settings, using simple content for objects to obtain the necessary digital content. However, complex applications use advanced recognition techniques and $A R$ applications to digitalize objects in a more precise and detailed way.

\subsection{Object Recognition Methods}

Various methods have been developed to recognize objects in AR applications. These methods are based on image processing, which is the principle of digitizing the image and converting it into a form that computers can understand. Target recognition is made by taking useful parts of the objects transferred to digital form. The useful parts have distinct details of the object. It is important to analyze the object well and choose a recognition method. In short, the details of the object inform us which recognition method will be chosen. In this study, three different object recognition methods and an advanced object recognition program are used.

In the early stages of the development of the AR application, it is planned to use the photographs of the objects to create target images. However, the fact that each element in SCADA panels needs to be photographed individually and has shown that the success rate of this recognition process will be affected negatively. Moreover, it has been found that a large number of photographs are required since the photography has to be taken from many angles. Instead of this approach, it is decided to photograph each of the devices on the panels (Energy quality recorder, W-automat, RTU, modem, ...) independently, regardless of the SCADA cabinet and panels.

For this purpose, Vuforia program is used as an object recognition program in the $\mathrm{AR}$ application which is proposed to be designed. Vuforia is a library that provides the ability to recognize images or objects through image processing technology and develop AR applications according to the location of the objects in the real world. Objects located in the real world can be identified using the three different object recognition methods discussed below.

\subsubsection{Image Target}

In this method, the photo of the target object is taken and digitalized. The captured image is loaded into the object recognition program database. It is desired that the uploaded images have planar features that provide sufficient detail that can be detected by the program. Images do not need to be divided into special black-and-white zones or contain conventional reference marks, such as quick response codes (QR). The image is graded by analyzing the natural features of the image. The rating is to show the detection level of the object and its traceability by the system. An image without enough detail and pattern is graded with a low score, while an image with different shapes, texts, characters, and patterns that do not repeat itself is rated with a high score. Other recognition methods are tried for objects with low grading scores. In Figure 1, images of two different objects graded with low and high scores are given.

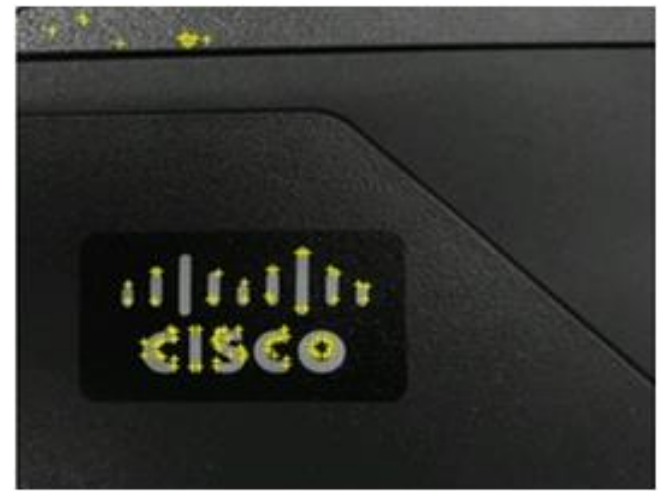

a)

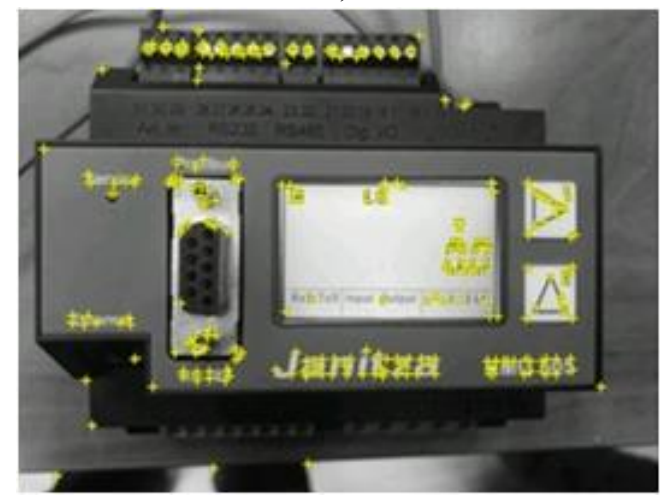

b)

Figure 1. Rating image target a) Low-scored image b) High-scored image.

For the image target method, a database must first be created within the Vuforia application. There are three different database types of Vuforia, which are specified as device, cloud, and vumark. These databases are shown in Figure 2 by taking a screenshot of Vuforia.

After selecting a suitable database for the application, the image target we want to recognize is loaded into the created database. There are different types of image targets that Vuforia offers to the user for object recognition. The target selection screen for two-dimensional objects, 3D cubic objects, 3D cylindrical objects, and other 3D objects that do not comply with this classification is shown in Figure 3. Once the loading process of the image target is finished, the rating of the image target can be seen as in Figure 1. After this process, the database created by Vuforia becomes available in the project where the application is developed. 


\subsubsection{Object Scan}

The method, which is used to recognize objects that are not very large, is for situations where the image target method is insufficient. The proposed study is used primarily to recognize the objects that can be scanned. Real objects are scanned and digitized with an application installed on mobile devices. Digital data is loaded into the database of the object recognition program, as in the image target method, and the objects are recognized. Scanning is carried out by rotating 360 degrees around the object. Thus, recognizing the target from different angles is presented.

For the object scan method, the three-dimension object scanner application running on the Android operating system within Vuforia is used. By running the application, the object placed on the image is scanned. After the scanning process is completed, the created file is loaded into the database by selecting the "3D Object" model. Figure 4 shows the RTU digitalization used in SCADA panels by the object scan method.

\subsubsection{Model Target}

Real objects are not used in this recognition method. 3D models of objects drawn using computer-aided design (CAD) programs are used. The 3D model is loaded into a converter program and converted into a format that the object recognition program can understand. In short, digital data is transformed into another form of digital data. This transformation program gives us an output showing the external lines of the object. In practice, when these lines are overlapped on the real object, the recognition process takes place. This method can be used for objects that do not have sufficient detail on their surface, do not contain patterns, and have CAD drawings. The size of the object is not important in this method. Even a car with a 3D CAD drawing can be easily recognized by this method.

Vuforia includes a program that enables the recognition of $3 \mathrm{D}$ drawing models in order to create a model target. The CAD drawing in the supported extension is transferred into the project by running this program, which is called the model target generator. After the CAD model object is transferred into the project, the angle we want to recognize the object is determined and the project output of the target file is saved in the computer. Figure 5 shows a visual of this process.

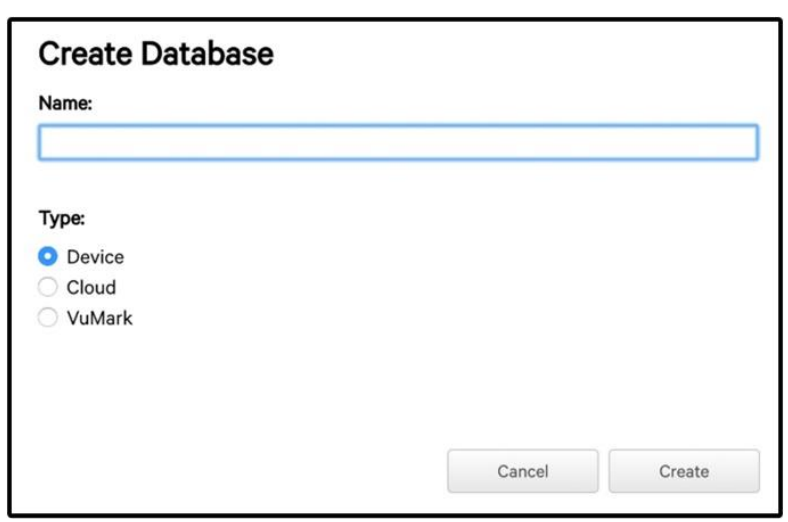

Figure 2. Creating a database in Vuforia.

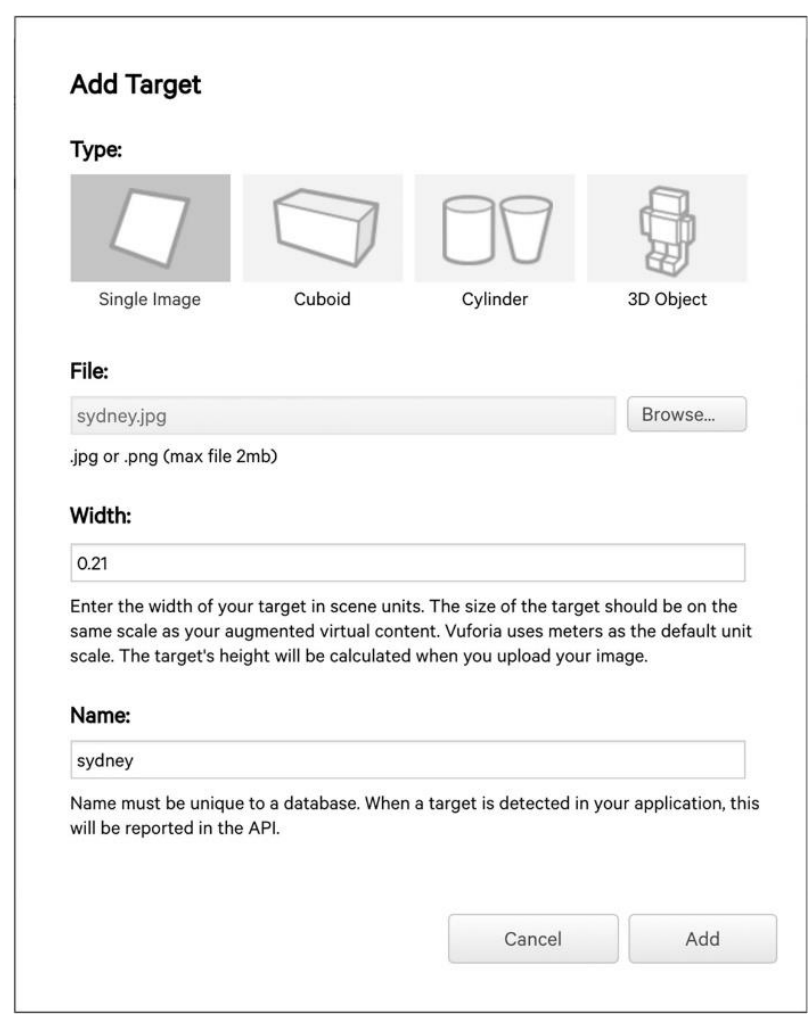

Figure 3. Adding image target in Vuforia.

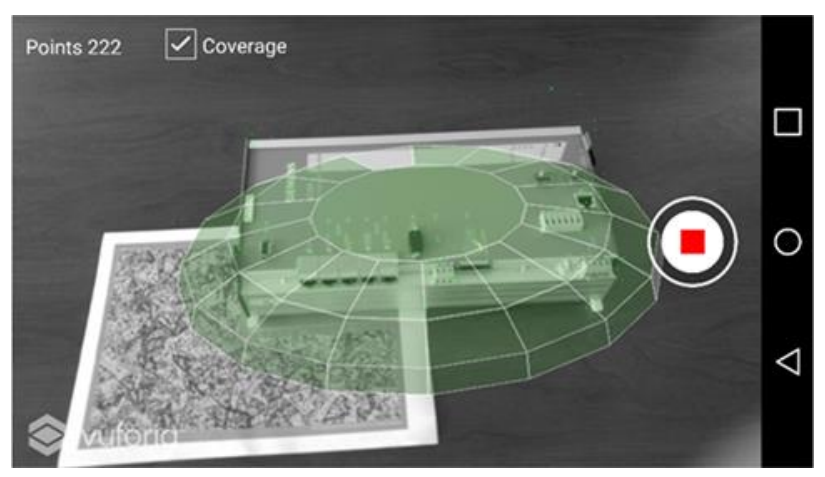

Figure 4. Object scanning for an RTU. 


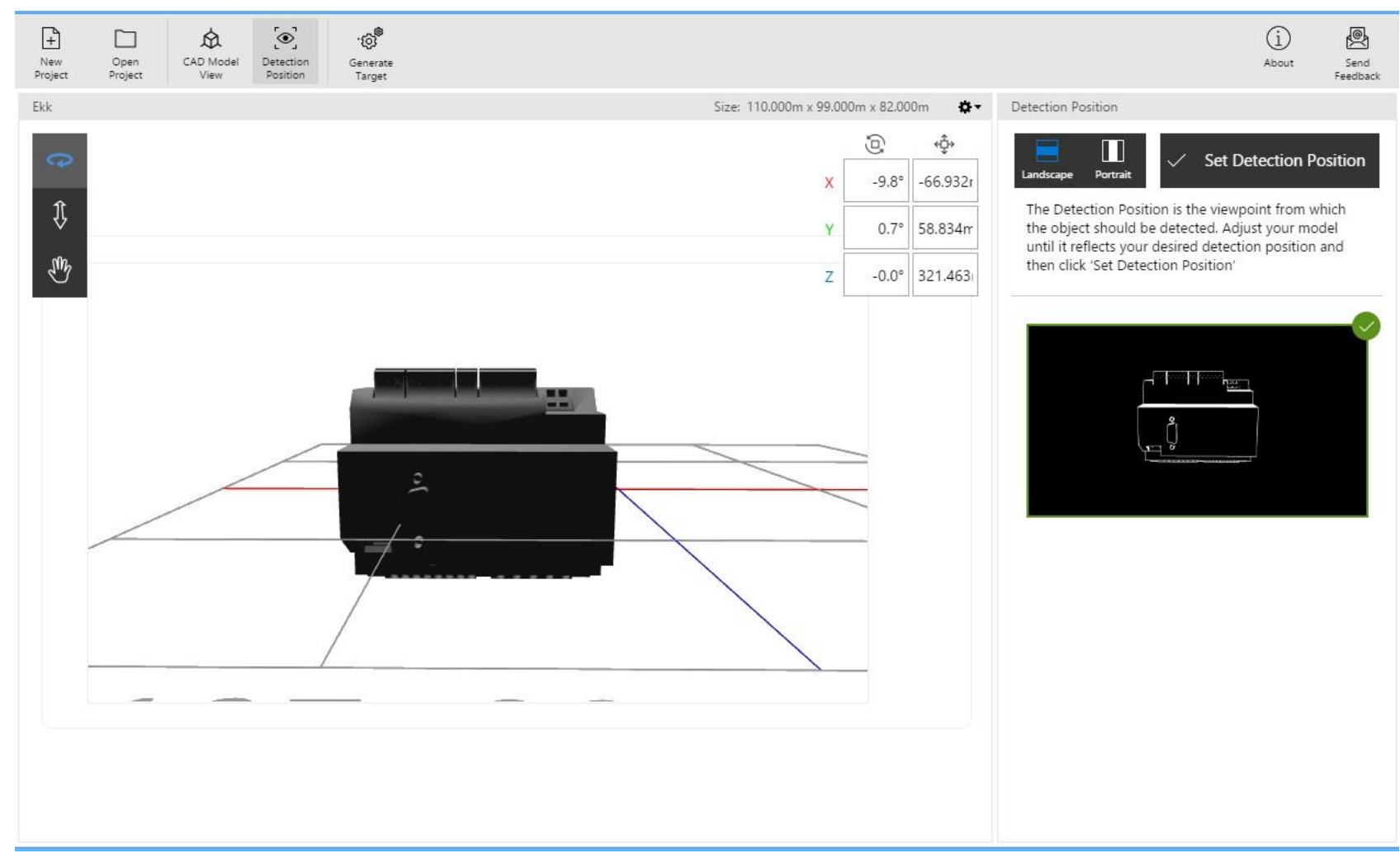

Figure 5. Object recognition with $\mathrm{CAD}$ drawing.

\subsection{AR Development Tool}

In the proposed study, ARKit software is used, which enables the determined objects that are detected and the AR processes start when the camera captures the objects. The library, called Object Scanner in the software, scans 360 degrees of the reference object placed on a flat surface. Once the scanning process is completed, the file with the extension ".arobject" is placed in the project in which the
AR application is developed in order to find the scanned object. The steps of this process performed for the energy quality analyzer (EQA) located in the panels in the SCADA center are shown in Figure 6. Objects in the project file are recognized by the relevant library through the Image Tracking module within ARKit, and reference objects are tracked as long as they are in the camera image. By using this module, horizontal-vertical planes and identified objects can be recognized quickly.

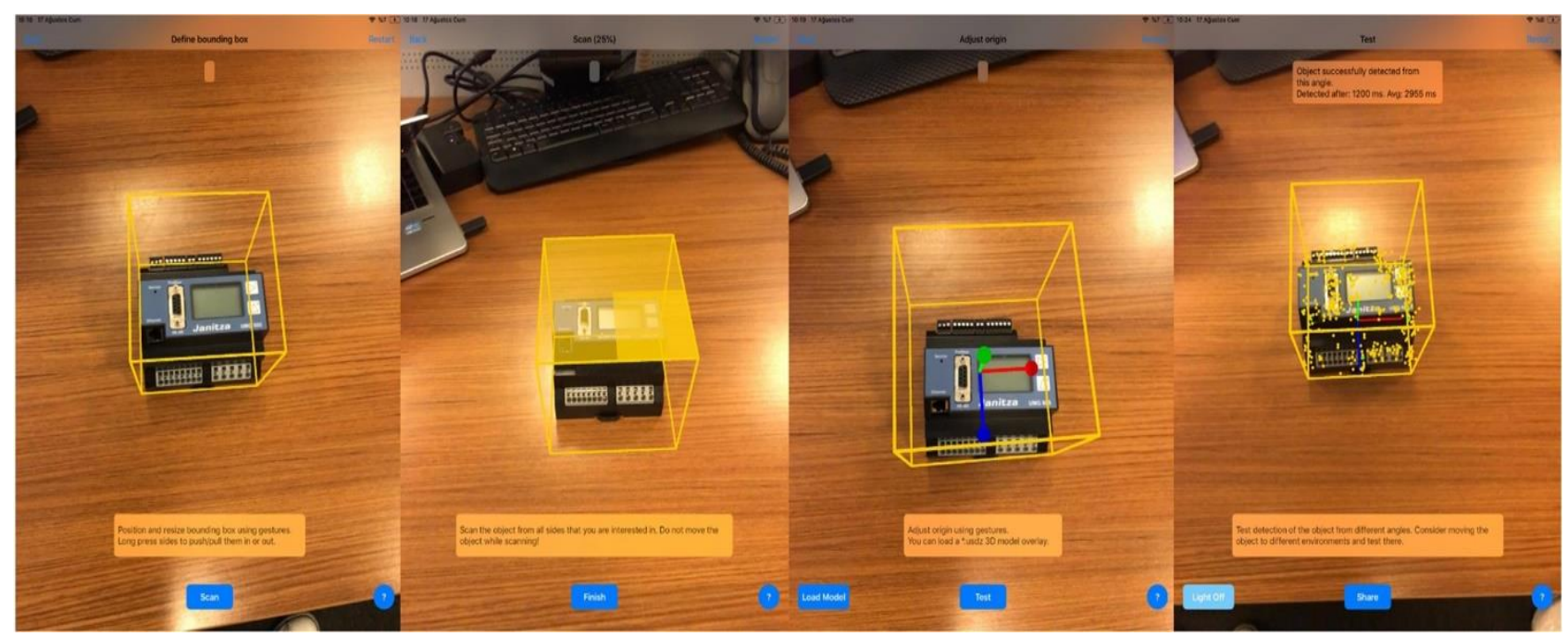

Figure 6. Scanning EQA. 


\subsection{Server and Database}

The AR application actually does not need a web server and can run independently on a device (tablet, phone). However, it requires a webserver to collect and report the valuable data generated on the device in a central database. In addition, the user name and password information to be used by the people who will use the AR application to $\log$ in to the application are also defined through the web interface. This information is sent to devices via web services. Therefore, the AR application needs a web server to be able to exchange data with a central database and generate reports.

In the proposed study, two databases are created. First case, when there is no communication network available, the system produces data over the device database. In the presence of a communication network, the system design is made in such a way that the data stored on the device are transmitted to the central database. Data are written to the device database after the user action. Buttons are shown to the user at each step. When these buttons are clicked, data are stored in the device database. Second case, when the problem can not be solved, and the information about the fault is stored on the device database. Therefore, when the communication network is provided, the information starts transmitting to the central database.

\section{Results and Discussion}

The facts that the department of SCADA development/maintenance (DM) team in SEDAS, headquartered in Gebze, has to travel in a wide area covering Kocaeli-Bolu-Düzce-Sakarya provinces for automation panel faults in SCADA cabinets od distribution centers with telemetry system, and the field maintenance teams do not have the experience to fix these faults are the most important starting point of the AR application proposed in this study. A system software has been implemented to work on AR technology-based mobile platforms that can take action more quickly and safely in order to solve possible problems that may occur in automation panels other than routine maintenance activities, reduce of occupational accidents, shorten of personnel hired adaptation period, minimize operational errors and raise the rate of resource utilization. A flowchart is used to create software is shown in Figure 7

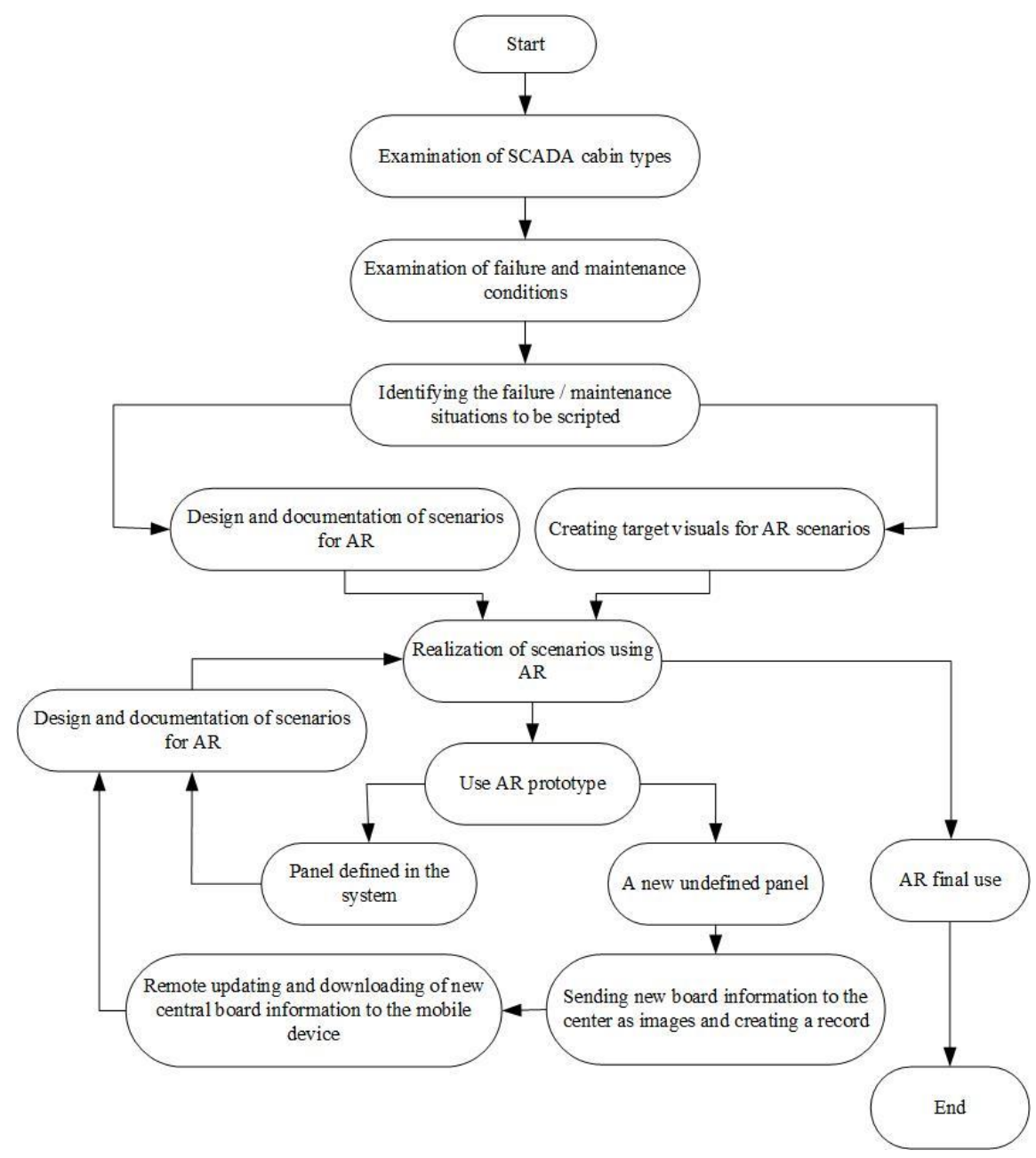

Figure 7. The roadmap of developed AR application. 
In the first stage, panels in SCADA cabinets are examined and MR scenarios are created for possible faults in these panels. A workflow scenario is drawn for each case. By analyzing the AR requirements of the Android platform on which the software environment is developed, the visuals are used to determine the SCADA cabinet which has been made recognizable by the application.

In the next step, screen designs are made for possible failure scenarios individually that may occur in the RTU and DCU automation panels of distribution centers. Scenarios are recorded for each step. Scenario steps, screen messages, buttons on the screen, and information about the next screen display can be defined systematically in this way. "RTU/IOBOX Automat Tripped", "No EQA Communication", "RTU EQA W-AUTO Control", "Rectifier Mainboard Replacement", "Rectifier AC Failure", "Rectifier Fan Failure", "No Communication", "RTU Module Failure" and "On/Off Failure" states are the names of the scenarios whose screen designs are realized. As an example, the screenshots designed for the "Rectifier Motherboard Replacement" scenarios are shown together in Figure 8.

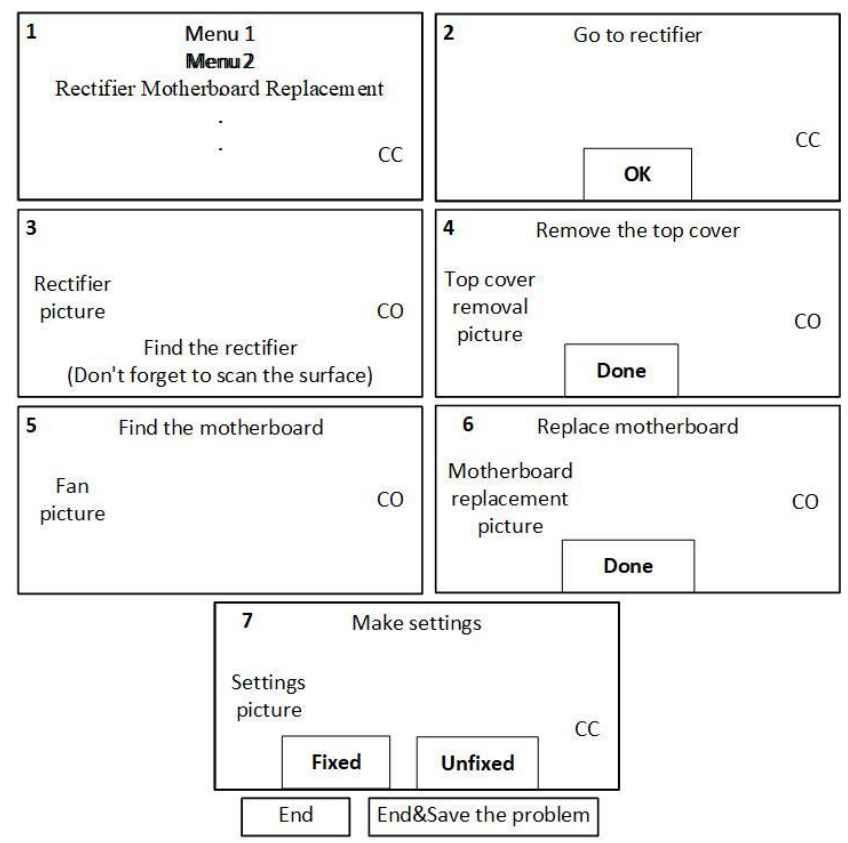

Figure 8. Rectifier motherboard replacement.

Two different recording procedures are made during the operation of the system. In every step of the application, during the interaction with the operator, the recording process is made and information such as the work number, scenario number, and time spent at each step, the buttons pressed at each step are kept. In this way, reports can be made on which scenarios work more (frequency of the problem), which steps are spent longer, and which problem the faults are mostly caused. In cases where the existing fault cannot be solved, records are stored to find out why the problem could not be solved.

In addition, a scenario record has been created for each scenario. The steps of each scenario, the messages and buttons that will appear on the screen, and the next step information when each button is pressed can be systematically defined in this way. The scenarios in the application are operated over ".json" extension files. The code text in this file is collected under two basic tags as "Scenario" and "Steps". In Figure 9, there is a part of the codes belonging to the ".json" file created for the "No Communication with EQA" scenario.

The scenario tag holds the information of the scenario name and scenario number. The step tag, on the other hand, holds the data used in the operation of the scenario steps. These data include the index used to access the sequence information in the series, step number, work order to be made, work order detail, whether the AR camera will be operated or not, the button to be displayed to the user in the work step, the number of the next work steps to go when the relevant button is clicked, and other scenarios information if there is any. Information obtained from these tags is reflected on user screens.

\begin{tabular}{|c|c|c|c|}
\hline 1 & \{ & 29 & \{ \\
\hline 2 & "Scenaria": \{ & 30 & "Index": 1, \\
\hline 3 & "Title":"EKK Haberleşme Yok", & 31 & "No":"2", \\
\hline 4 & "No":2, & 32 & "Title":"Hücre Yüzeyini Tara", \\
\hline 5 & "Index":1 & 33 & "Description":"", \\
\hline 6 & \} & 34 & "TrackImage":false, \\
\hline 7 & "Steps":[ & 35 & "TrackImageName":null, \\
\hline 8 & \{ & 36 & "Input":[ \\
\hline 9 & "Index": 0 , & 37 & \{ \\
\hline 10 & "No":"1", & 38 & "Item":"BtnOk", \\
\hline 11 & "Title":"Hücreye Git Kapağ1 Aç", & 39 & "NextStep":2 \\
\hline 12 & "Description":"", & 40 & \}, \\
\hline 13 & "TrackImage":false, & 41 & \{ \\
\hline 14 & "TrackImageName":"", & 42 & "Item":"BtnBack", \\
\hline 15 & "Input":[ & 43 & "NextStep":999 \\
\hline 16 & \{ & 44 & \} \\
\hline 17 & "Item":"BtnOk", & 45 & ], \\
\hline 18 & "NextStep": 1 & 46 & "NextScenaria":null, \\
\hline 19 & \} & 47 & "Media":true, \\
\hline 20 & ], & 48 & "Source":null, \\
\hline 21 & "NextScenaria":null, & 49 & "Animation":null, \\
\hline 22 & "Media":true, & 50 & "TriggerName":null, \\
\hline 23 & "Source":null, & 51 & "RekranMain":"11", \\
\hline 24 & "Animation":null, & 52 & "RekranSub":"22" \\
\hline 25 & "TriggerName":null, & 53 & \} \\
\hline 26 & "RekranMain":"1", & & \\
\hline 27 & "RekranSub":"2" & & \\
\hline 28 & \}, & & \\
\hline
\end{tabular}

Figure 9. The code for the first two steps of the ".json" file created for "No EQA Communication" scenario.

In Figure 10, a rectifier and RTU-DCU automation panels, as well as EQA used in these panels, can be seen in the photograph of a distribution center within SEDAS.

The developed AR application is used on the EQA device in this distribution center, and the scenario of "No EQA Communication" is executed. The EQA image obtained from the mobile device camera used by the operator is shown in Figure 11. Figure 12 shows some of the photographs of the scenario steps obtained when the "No EQA Communication" scenario is started for the device detected by the camera. 


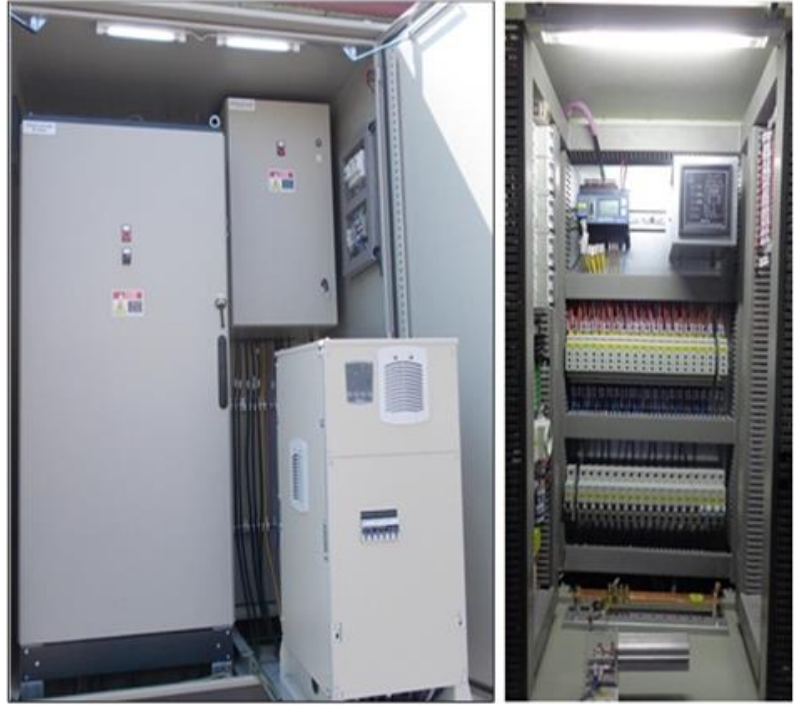

Figure 10. Automation panels of distribution center a) RTU, DCU and rectifier b) EQA in the panel.

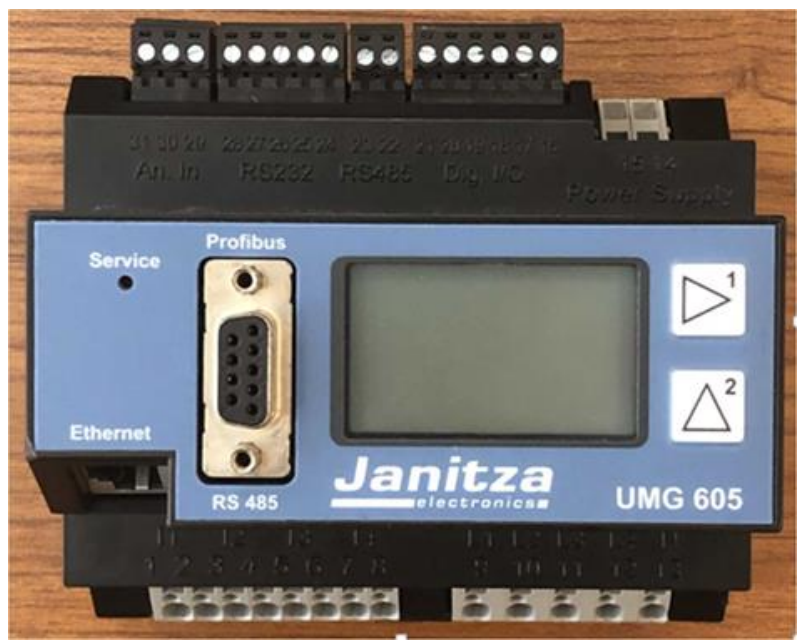

Figure 11. EQA image obtained from mobile camera.

The operator switches between scenario steps by following the instructions specified in the scenario according to the fault and terminates the process. When the fault is fixed, the operations performed at each step are recorded with the "Finish/Save" button. On the other hand, when the fault remains unfixed, then this situation is recorded with the "Finish/Save Problem" button.

\section{Conclusions}

AR is an innovative technology that has the potential to be applied in many different industries. In parallel with the increase in processor capacities in mobile devices and the image quality of cameras and the introduction of smart glasses and wearable AR systems for the end-user, it is predicted that AR applications will be used frequently in the electrical energy sector in the near future, as in other sectors.
Therefore, electrical energy suppliers also need to adapt to AR technology as quickly as possible in order not to lose their competitiveness in the market.

In the proposed study, a Software that works on AR technology-based mobile platforms is developed and used in maintenance and repair processes on protection/control panels and devices in SCADA centers, apart from routine maintenance activities. The main aim of using this Software is to provide task support by quickly detecting faults to field maintenance teams. For this purpose, a software application is implemented for possible fault scenarios that may occur in RTU and DCU automation panels of SCADA cabinets located in distribution centers. The gains from this application that also contributes to digital transformation can be listed as follows:

The fact that the teams of DM Department within SEDAS traveled multiple times to the distribution center where the defective cabin is located for detecting faults and MR operations in the SCADA cabins in the area of responsibility covering four provinces, causing time and budget losses. The ability to have these operations performed by field teams, thanks to the developed software application has minimized the losses. Thus, it is predicted to save approximately 50.000 TL per year. At the same time, with reduced fuel consumption, approximately 7 tons of carbon dioxide emission per year will be prevented and it will contribute to the reduction of the carbon footprint.

As the workload of the staff is reduced in the DM Department, which consists of an expert team, pending jobs can be handled faster.

From the perspective of occupational safety, it is guaranteed to reduce the exposure of expert personnel to risks during long journeys. According to the risk assessment, the risk value, which is at the significant risk level, has been reduced from 600 to 150 .

Thanks to the developed mobile application, human errors are reduced by following the steps that should be taken to eliminate the failure.

A backup facility, albeit partially, has been created expert personnel and a training portal has been provided for new recruits in the department.

The proposed study constitutes an example of the AR technology applications, which is rare in the electricity energy sector both in our country and in the world.

In short, the advantages of AR technology-based applications are they can be easily used in information transfer. They are highly productive, they have increased efficiency with a low error rate, high quality, and safety. The procedures are simplified, they have effective resource usage and capacity of early adoption of learning that makes the use of this innovative technology important. 

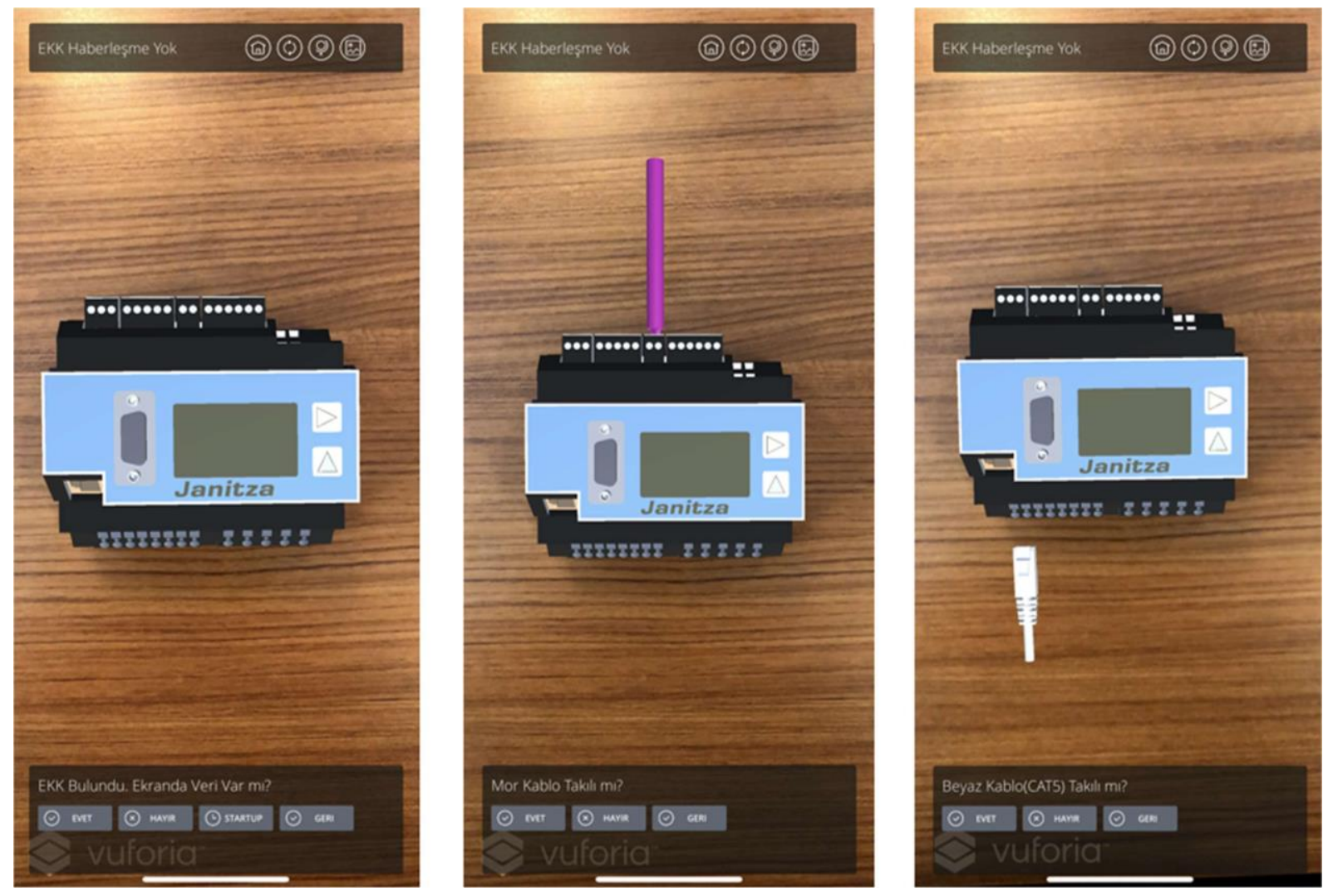

Figure 12. Some of the images of the "No EQA Communication" scenario steps.

\section{Declaration of Ethical Standards}

The authors of this article declare that the materials and methods used in this study do not require ethical committee permission and/or legal-special permission.

\section{Conflict of Interest}

The authors declare that they have no known competing financial interests or personal relationships that could have appeared to influence the work reported in this paper.

\section{Acknowledgements}

This study was supported by the "Maintenance-Repair and Training Applications in SCADA Operations with Augmented Reality Technology" project by EMRA.

\section{References}

[1] Porter M.E., Heppelmann J.E., 2017. Why Every Organization Needs an Augmented Reality Strategy. Harvard Business Review Magazine, 95(6), 46-57.

[2] AccuVein: https://www.accuvein.com/whyaccuvein/ar, (05.03.2019)
[3] Boeing Tests Augmented Reality in the Factory: https://www.boeing.com/features/2018/01/augmented -reality-01-18.page, (07.07.2017)

[4] Hastings E.J., Guha R.K., Stanley K.O., 2009. Automatic Content Generation in the Galactic Arms Race Video Game. IEEE Transactions on Computational Intelligence and AI In Games, 1(4), 245-263.

[5] Matsutomo S., Miyauchi T., Noguchi S., Yamashita H., 2012. Real-Time Visualization System of Magnetic Field Utilizing Augmented Reality Technology for Education. IEEE Transactions on Magnetics, 48(2), 531-534.

[6] Sheng Y., Yapo T.C., Young C., Cutler B., 2011. A Spatially Augmented Reality Sketching Interface for Architectural Daylighting Design. IEEE Transactions on Visualization and Computer Graphics, 17(1), 3850 .

[7] Sirilak S., Muneesawang P., 2018. A New Procedure for Advancing Telemedicine Using the HoloLens. IEEE Access, 6, 60224-60233.

[8] Fraga-Lamas P., Fernández-Caramés T.M., BlancoNovoa Ó., Vilar-Montesinos M.A., 2018. A Review on Industrial Augmented Reality Systems for the Industry 4.0 Shipyard. IEEE Access, 6, 13358-13375. 
[9] Cheng K.H., Tsai C.C., 2013. Affordances of Augmented Reality in Science Learning: Suggestions for future search. Journal of Science Education and Technology, 22(4), 449-462.

[10] Rosenbaum E., Klopfer E., Perry J., 2007. On Location Learning: Authentic Applied Science with Networked Augmented Realities. Journal of Science Education and Technology, 16(1), 31-45.

[11] Pérez-López D., Contero M., 2013. Delivering Educational Multimedia Contents Through an Augmented Reality Application: A Case Study on Its Impact on Knowledge Acquisition and Retention. The Turkish Online Journal of Educational Technology, 12(4), 19-28.

[12] Parmar D., Pelmahale K., Kothwade R., Badgujar P., 2015. Augmented Reality System for Engineering Graphics. International Journal of Advanced Research in Computer and Communication Engineering, 4(10), 327-330.

[13] Bozyer Z.,2015. Augmented Reality in Sports: Today and Tomorrow. International Journal of Science Culture and Sport, 3(4), 314-325.

[14] Serino M., Cordrey K., McLaughlin L., Milanaik R.L., 2016. "Pokemon Go and Augmented Virtual Reality Games: A Cautionary Commentary for Parents and Pediatricians. Current Opinion in Pediatrics, 28(5), 673-677.

[15] Arroyo E., Arcos J.L.L., 1999. SRV: A Virtual Reality Application to Electrical Substations Operation Training. Proceedings IEEE International Conference on Multimedia Computing and Systems, 835-839.
[16] Arendarski B., Termath W., Mecking P., 2008. Maintenance of Complex Machines in Electric Power Systems Using Virtual Reality Techniques. Conference Record of the 2008 IEEE International Symposium on Electrical Insulation, 483-487.

[17] Cardoso A., et al., 2013. VRCEMIG: A Virtual Reality System for Real Time Control of Electric Substations. 2013 IEEE Virtual Reality (VR), 165-166.

[18] Hernández Y., Ramírez M.P., 2016. Virtual Reality Systems for Training Improvement in Electrical Distribution Substations. 2016 IEEE 16th International Conference on Advanced Learning Technologies, 75-76.

[19] Peng Y., Yu G., Ni W., Lv Z., Jiang Y., Chen J., 2017. Design and Development of Intelligent Operation and Maintenance Training System for Substation Based on Augmented Reality. 2017 Chinese Automation Congress, 4765-4769.

[20] Cordonnier M., et al., 2017. Contribution of Augmented Reality to the Maintenance of Network Equipment, International Conference on Electricity Distribution Open Access Proceedings Journal, 1, 8790.

[21] Martino S., Gonbeau O., Boisseau C., Recapet J., Blanc F., Augustin B., 2017. Enedis Field Experience of Augmented and Virtual-Reality Technologies at the Service of Network Operators. International Conference on Electricity Distribution Open Access Proceedings Journal, 1, 1081-1084.

[22] Sermet M.Y., Demir I., Kucuksari S., 2018. Overhead Power Line Sag Monitoring through Augmented Reality. North American Power Symposium, 1-5. 